\title{
Circadian disruption and cancer risk: A new concept of stromal niche (Review)
}

\author{
HIROTO IZUMI $^{1}$, KEYONG WANG ${ }^{2}$, YASUO MORIMOTO ${ }^{1}$, YASUYUKI SASAGURI ${ }^{3}$ and KIMITOSHI KOHNO ${ }^{4}$ \\ ${ }^{1}$ Department of Occupational Pneumology, Institute of Industrial Ecological Science, ${ }^{2}$ Bio-information Research Center, \\ ${ }^{3}$ Center for Promotion of Clinical and Translational Research, ${ }^{4}$ The President Laboratory, \\ University of Occupational and Environmental Health, Kitakyushu, Fukuoka, Japan
}

Received September 19, 2013; Accepted October 29, 2013

DOI: 10.3892/ijo.2013.2201

\begin{abstract}
Circadian rhythms regulate a variety of physiological functions. Epidemiological evidence indicates that disruption of these circadian rhythms might be linked to cancer. In general, imbalances in homeostasis, such as immune and hormonal dysfunctions, are thought to be involved in cancer development. The results of a recent study suggested that circadian disruption may induce stromal changes associated with cancer risk, highlighting the importance of the cancer stem cell niche for protecting cancer cells. Current research provides new concepts and clarification regarding the function of the tumor niche, and the new concept of a stromal niche may help us to understand the additional functions of both cancer-associated fibroblasts and the extracellular matrix. In this review, we summarize our current knowledge regarding the role of circadian rhythms in cancer risk and the relevance of the stromal niche in cancer cell survival and progression.
\end{abstract}

\section{Contents}

1. Epidemiological evidence

2. Cancer stem cell niche

3. Niche-driven tumor progression

4. Cancer-associated fibroblasts and tumor microenvironment

5. Tumor-driven niche development

6. Drug resistance

7. Conclusions

Correspondence to: Professor Kimitoshi Kohno, University of Occupational and Environmental Health, 1-1 Iseigaoka Yahatanishi-ku, Kitakyushu City, Fukuoka 807-8555, Japan

E-mail:k-kohno@med.uoeh-u.ac.jp

Key words: circadian disruption, WNT10A, stromal niche, cancer stem cell, cancer-associated fibroblast

\section{Epidemiological evidence}

The use of electric lights means that people in the modern era are exposed to long photoperiods throughout the year. This disruption to the circadian system can induce a wide variety of stresses. Abnormal circadian rhythms, including through exposure to light at night, have been associated with an increased risk of tumorigenesis and a poorer prognosis in carcinomas $(1,2)$. This suggests that the incidence of cancer may continue to increase, in association with the stresses of modern life. The risks of both breast and prostate cancer are high in industrialized societies (3). Continuous hormonal unbalances in shift workers might be caused by circadian disruption and may contribute to the rising risk of cancer. Endocrine target organs, such as the breast and prostate, are thought to be susceptible to psychosocial stresses, including circadian disruption (4). The immunological surveillance system has also been shown to be affected, and may thus not eliminate cancer stem cells (CSCs) effectively (5).

Circadian genes have been shown to function as oncogenes or tumor suppressors at systemic or cellular levels through their involvement in cell proliferation, cell cycle regulation, apoptosis and DNA damage signaling $(6,7)$, indicating a direct effect of circadian effects on cancer cells. However, the kinds of molecular and systemic mechanisms involved in tumor growth under artificial illumination stress remain unknown, and the importance of artificial illumination in promoting tumor growth also needs to be established. We propose an indirect mechanism supporting the survival of potential CSCs and discuss a new concept of tumor niche formation induced by circadian disruption.

\section{Cancer stem cell niche}

Clinical tumors comprise a heterogeneous cell population including CSCs (8). The malignant phenotype depends not only on the characteristics of the cancer cell itself, but also on the tumor microenvironment. CSCs have to survive for a long time in the body to generate the highly tumorigenic cells responsible for the clinical manifestations of cancer. During this period, the niche helps to shelter CSCs from various insults such as the immune response, and from genotoxic stresses such as chemotherapy $(9,10)$. This suggests that the niche may also play a protective role for CSCs, and may thus contribute to the risk of cancer. 


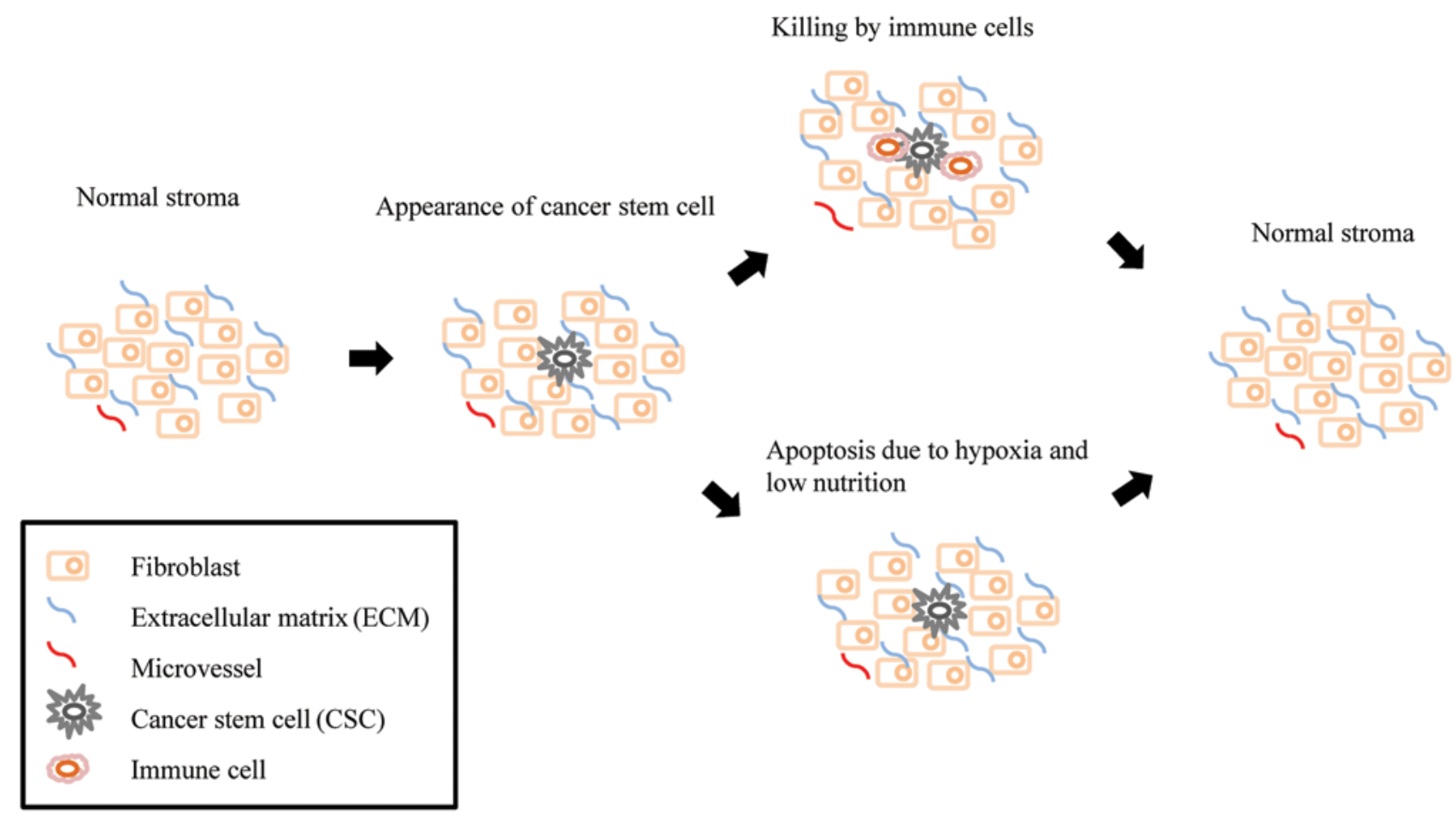

Figure 1. Cancer stem cells in normal stroma.

\section{Niche-driven tumor progression}

Although CSCs appear relatively frequently, they are unable to survive in the healthy body without supportive tissues. As shown in Fig. 1, CSCs are killed by immune cells, or disappear under conditions of hypoxia and malnutrition. In general, CSCs only survive in the primary niche long enough to cause cancer when the microenvironment is supportive. The primary niche is composed of fibroblasts and the extracellular matrix (ECM) (11). Circadian disruption activates the fibroblasts, which produce the autocrine growth factor, WNT10a. These WNT10a-producing fibroblasts secret ECM, and may provide the beneficial conditions required to form an initial tumor niche or microenvironment for CSCs (Fig. 2). It is possible that this process may contribute to the maturation of CSCs, but not their differentiation. Psychosocial stress might activate resident fibroblasts in the body, which together with the ECM, may provide the niche required for CSCs in the preclinical phase. Mouse NIH3T3 cells overexpressing WNT10a can grow rapidly and form tumors in nude mice. As shown in Fig. 3, WNT10a-producing cells promote their own growth and secrete ECM. Interestingly, it has been reported that increased collagen density promotes mammary tumor initiation and progression (12).

It has been proposed that metastasis requires the existence of a metastatic niche to allow the invading cancer cells to survive, colonize and expand to form macrometastases (13). It has been reported that both cancer cells and cancer-associated fibroblasts (CAFs) produce angiogenic factors, such as vascular endothelial growth factor and WNT10a, respectively, while it is well known that cancer cells produce reactive oxygen species (ROS) that induce these angiogenic factors (14). Rapid tumor growth with angiogenesis induces extracellular acidosis, which in turn leads to activation of metalloproteinases that destroy the structure of the tumor niche (15). Hypoxic glycolysis is activated and produces acid metabolites, and the subsequent decrease in intracellular $\mathrm{pH}$ has been shown to reduce DNA repair activity, resulting in the accumulation of spontaneous mutations following the malignant progression of cancer cells. Thus, solid tumors finally disrupt the niche through degradation of the ECM, and acquire the ability to invade tissues and to metastasize during the clinical phase. The tumor microenvironment is thus crucial for solid tumor development (16), and dysregulation of the $\mathrm{pH}$ and disruption of the tumor niche is closely involved in the cancer hallmarks of invasion and metastasis. These observations also suggest that circadian disruption in cancer patients might be related to poor prognosis.

\section{Cancer-associated fibroblasts and tumor micro- environment}

Fibroblasts are an abundant cell type in connective tissues. They produce ECM components and various cytokines, and contribute to the formation of a structural network through tissue remodeling (17). The tumor environment includes structural and cellular components. The cellular components are the so-called stromal cells, including both resident and circulating cells such as macrophages, inflammatory cells, endothelial cells, myofibroblasts and CAFs (18). Increasing evidence indicates that CAFs are a main player in the hallmarks of cancer such as angiogenesis, invasion, metastasis and inflammation, which are critical factors for the development of solid tumors (Fig. 4). However, the origin of CAFs remains unknown (19). Mesenchymal stem cells contribute to the formation of tumor-associated stroma containing cellular components such as myofibroblasts and fibro- 


\section{Appearance of activated fibroblasts by stress such as circadian disruption}

\section{Formation of activated stroma \\ Formation of activated stroma
WNT10A-dependent autocrine growth of activated fibroblasts}

\section{grewh of activatedibroblasts}

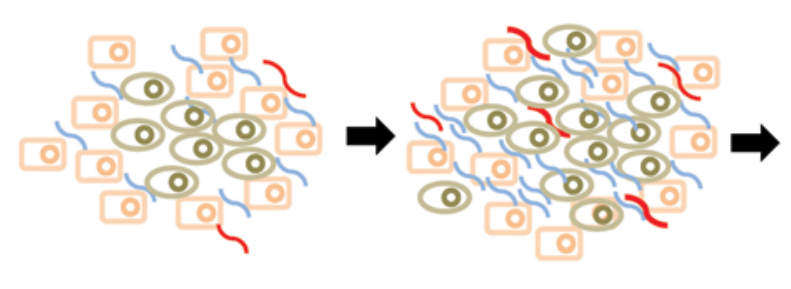

WNT10A-dependent increase of ECM and microvessels
Appearance of cancer stem cell

Cancer stem cell can survive under activated stroma

Figure 2. Cancer stem cells in stress-activated stroma.

Asymmetric division of cancer stem cell and appearance of cancer cells

\footnotetext{
(2) Activated fibroblast
2 WNT10A-dependent angiogenesis
to: Cancer cell
}

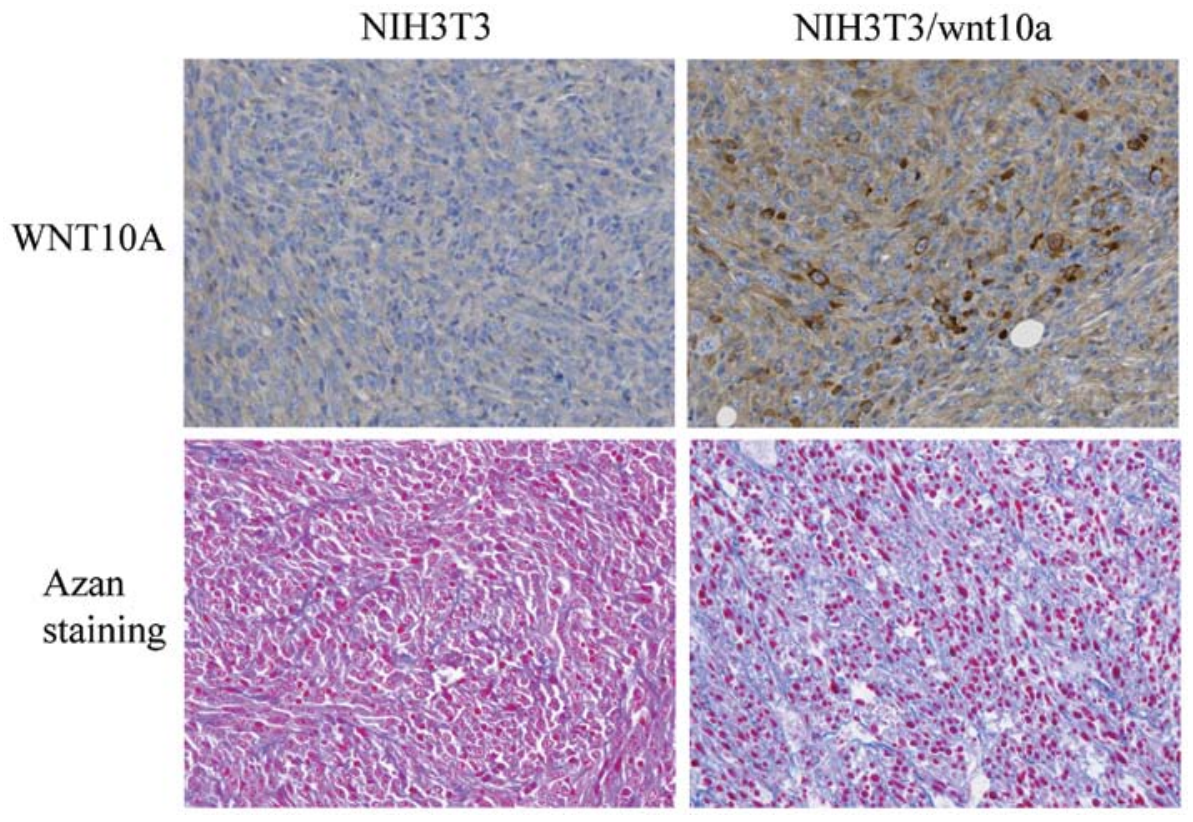

Figure 3. ECM deposition in WNT10A-overexpressing NIH3T3 tumors. Eight-week-old male nude mice (BALB/c nu/nu; Kyudo Co.) were used for subcutaneous xenografting. Mice were injected with $100 \mu \mathrm{l}\left(1 \times 10^{6}\right.$ cells) of WNT10A-overexpressing NIH3T3 cell suspension at two separate dorsal sites. Subcutaneous xenografting experiments were repeated three times. Formalin-fixed tumors were embedded in paraffin and sections were immunostained with anti-WNT10A (1:50, Sigma-Aldrich) according to the manufacturer's instructions. The anti-WNT10A antibody recognizes both human and mouse WNT10A. Azan staining was used to evaluate the extracellular matrix. All procedures were approved by the ethics committee of the University of Occupational and Environmental Health.

blasts (20-23). Furthermore, the detailed roles of CAFs are still unclear, and not all the cytokines produced by CAFs have yet been identified. It is possible that the properties of CAFs important during the early stage of tumor development differ from those involved in the late stage. CAFs may be activated by factors in the microenvironment, such as hypoxia (24), and by physi- ological conditions such as circadian rhythms. We demonstrated that disruption of circadian rhythms can promote tumor growth through WNT10A-dependent angio/stromagenesis, associated with increased levels of oxidative stress (25). Both endothelial cells and stromal cells may be activated by WNT10A signals from non-tumor cells such as CAFs. WNT signaling has been 
Cancer cell growth Appearance of CAFs

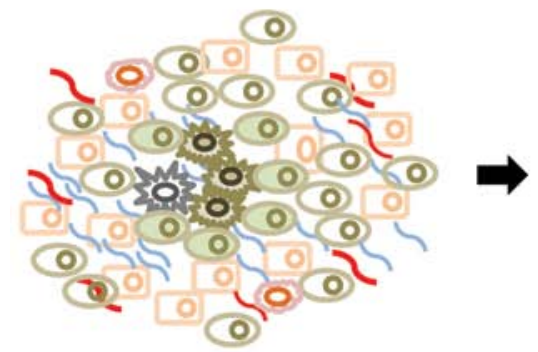

VEGF-dependent angiogenesis Appearance of TAMs
Solid tumor in clinical phase Invasion and metastasis following formation of angio/metastatic niche

\footnotetext{
Cancer associated fibroblast (CAF)
VEGF-dependent angiogenesis
Tumor associated macrophage (TAM)
}
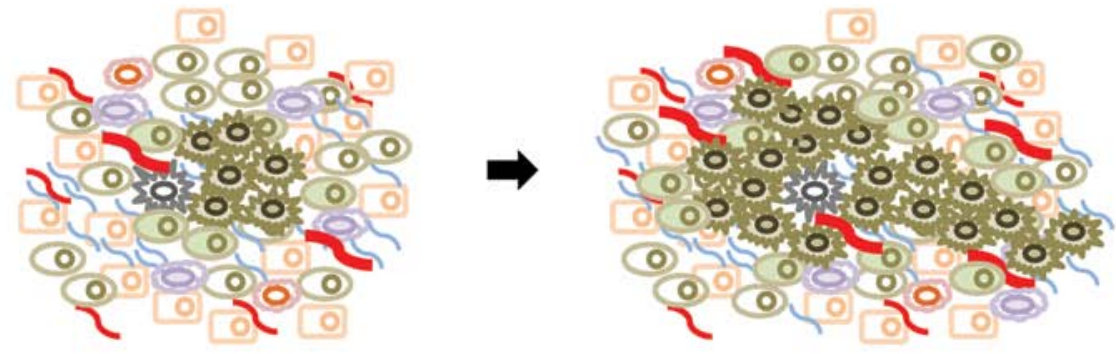

Figure 4. Solid tumor development with cancer-associated fibroblasts.

classified into 'canonical' and 'non-canonical' pathways. In addition, $\beta$-catenin expression has been observed in endothelial cells in newly-formed tumor vessels, suggesting that $\mathrm{WNT} / \beta$-catenin signaling plays a role in tumor angiogenesis. WNT signaling is also known to play an important role in cancer and stem cell biology (26), indicating that WNT10A might affect not only the tumor microenvironment, but also the CSCs themselves.

\section{Tumor-driven niche development}

The generation of CSCs and cancer progression involve a long-term and complicated series of processes. Accumulating evidence suggests that psychosocial stress can influence cancer cell growth via many processes. Cancer cells induce inflammation (27). Both tumor-associated macrophages and CAFs are critical for cancer progression (28). In addition, both cancer cells and activated stromal cells produce high levels of ROS and cytokines. ROS induce not only DNA damage following malignant transformation, but also CAF activation. These tumor-host interactions alter the local tumor environment and contribute to tumor growth. Activated CAFs produce ECM around cancer cells, and CAF-dependent ECM production may support the formation of a new niche allowing the development of local micrometastases around the primary tumor. Finally, the development of an angiogenic niche around the main tumor supports invasion and metastasis. Fig. 5 shows a typical case that supports this idea. Tumor-associated connective tissue is often observed in front of the main gastric tumor. Both azan staining and immunohistochemical studies showed that this region was rich in collagen and microvessels (Fig. 5B and C), and the vascular smooth muscle cells in the microvessels and the fibroblastic cells observed in the collagen deposits were positive for WNT10A (Fig. 5D). WNT10A may thus contribute to the formation of the angio/stromagenic niche. We also suggest that the tumor-associated connective tissue functions as an angio/stromagenic niche around the primary tumor. Bateman (29) also proposed that cancer cells modify the stroma of remote organs and create a premetastatic niche.

WNT10A mutations are associated with autosomal recessive ectodermal dysplasia (30). In addition, the expression of WNT signaling antagonists has been shown to be downregulated in fibroblasts in keloids, which are an aggressive type of wound-healing tissue (31). These previous reports indicate that WNT signaling is involved in both tissue repair and wound healing. An earlier hypothesis suggests that cancer results from uncontrolled wound-healing (32). This is supported by the observation that WNT10A expression was markedly increased in fibroblastic cells in the hyperplastic stroma of keloid tissue, suggesting its function as an angio/stromagenesis gene in tumor progression.

There are some limitations associated with experiments using mice. We cannot exclude the possibility that other physiological and/or hormonal factors, such as melatonin, may affect the growth of implanted cancer cells in mouse models. Melatonin suppression through exposure to artificial light at night leads to carcinogenesis of target endocrine organs (33). However, serum melatonin cannot be measured in mice because the pineal gland is too small. Thus although the subcutaneous injection of rapidly growing human cancer cells into nude mice provides a setting in which tumor growth can be assessed in a relatively short time span, an orthotopic model that more accurately reproduces the interactions between tumor cells and their microenvironment is required to confirm these results.

\section{Drug resistance}

The molecular mechanisms responsible for the cellular sensitivity to anticancer agents have been extensively studied in cancer cell lines (16). However, drug resistance is also influenced indirectly by the tumor microenvironment. Cisplatin resistance is affected by several factors that influence intracellular drug accumulation, including the levels of cellular thiols and DNA 

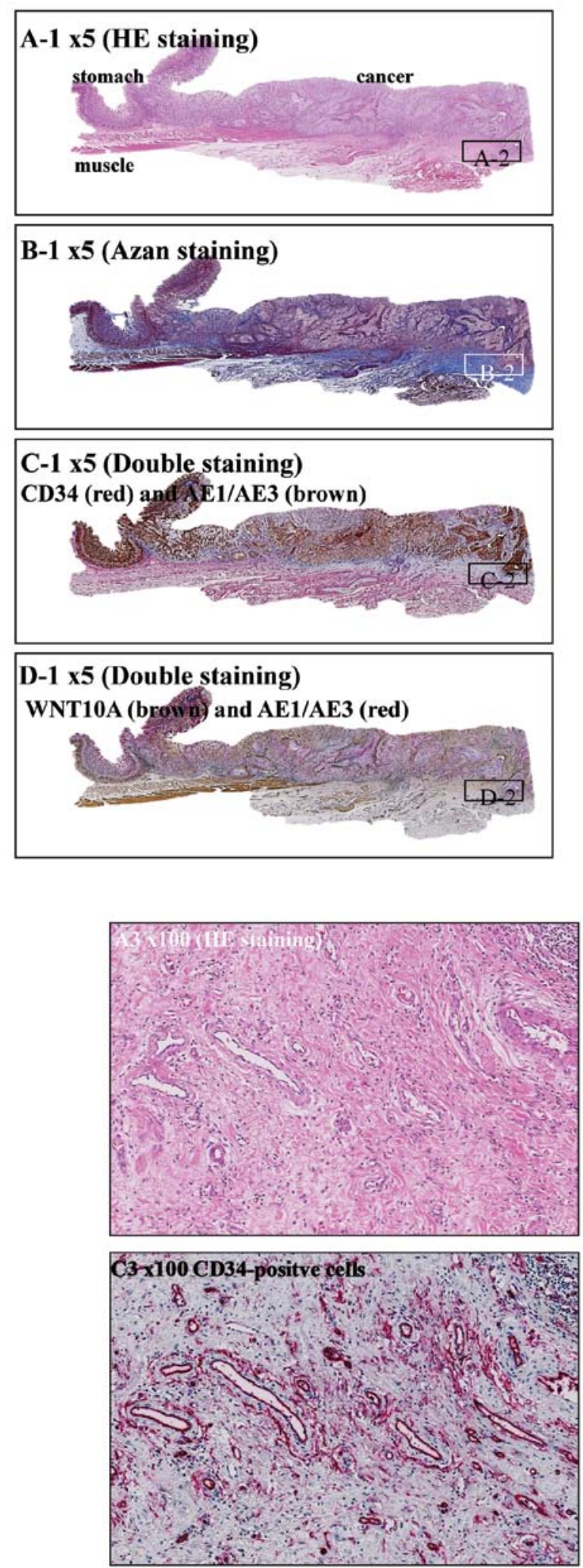
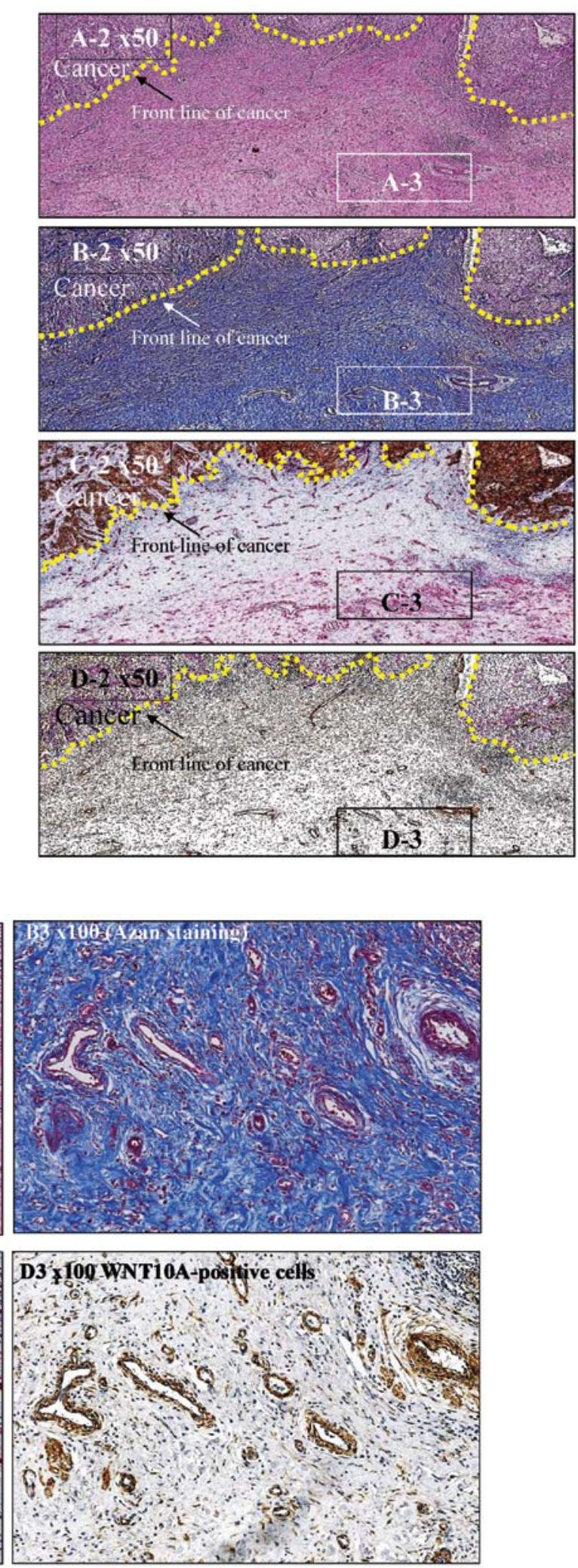

Figure 5. Stomach cancer with remodeling of connective tissue as an angio/stromagenic niche. (A) Hematoxylin and eosin stained squamous cell carcinoma at the gastro-esophageal junction. Tumor penetrates the serosa (A-1). Carcinoma arranged in sheet-like nests or irregular cords confined to the serosa. Yellow dotted line indicates the front line of the cancer partitioned from the stroma (A-2). Scar-like stromal reaction, in which peripheral desmoplasia with proliferating spindle-shaped cells and numerous well-developed blood capillaries, is found throughout the front line of carcinoma progression (A-3). (B) Azan staining. In desmoplastic stromal areas, spindle-shaped cells and blood capillaries were counterstained red (B-1 and 2), as were nuclei, fibrin and epithelial hyaline bodies. Collagen fibers and mucus were counterstained blue, together with the basal lamina of the blood capillaries (B-3). (C) CD34 (red) - AE1/AE3 (brown) double staining method. Immunohistochemically, carcinoma cells were positive for AE1/AE3 (brown) and angioendothelial cells were positive for CD34 (red) (C-1 and 2). In stromal areas, spindle-shaped cells and the basal lamina of blood capillaries were stained red (C-3). (D) AE1/AE3 (red) - WNT10 (brown) double staining method. Immunohistochemically, carcinoma cells were positive for AE1/AE3 (red) and the stromal area was positive for WNT10 (brown) (D-1 and 2). In the stromal area, spindle-shaped cells and the basal lamina of blood capillaries were stained brown (D-3). 


\title{
Circadian disruption, tumor niche and development of solid tumor
}

\author{
Psychosocial stress $\rightarrow$ Activated stromal niche with activation of fibroblasts \\ and ECM and cytokine production \\ WNT10A-dependent angio/stromagenesis \\ CSCs \\ $\sqrt{ }$ Malignant transformation \\ Continued proliferation \\ $\sqrt{1}$ \\ Malignant progression \\ Evade apoptosis
}

\section{Activated CAFs and TAMs}

\section{$\downarrow \quad$ VEGF-dependent angiogenesis}

Solid tumor development in clinical phase

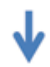

Invasion

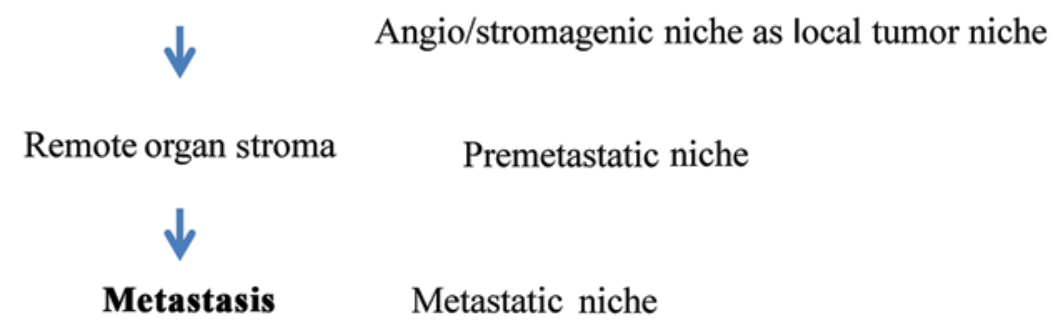

Figure 6. Schematic summary.

repair activity (34). Our own studies showed that glutathione biosynthesis was upregulated by activating transcription factor 4 (ATF4), which is also regulated by the circadian transcription system $(35,36)$. ATF4 expression was induced by oxidative stress through the Nrf2 transcription factor (37). The role of histone acetyltransferase (HAT) gene expression in the development of drug resistance has not been extensively studied, though it has been shown that HAT genes such as CLOCK and TIP60 are overexpressed, and are involved in glutathione biosynthesis and DNA repair (38), indicating that the system protecting against various stresses might be regulated periodically. These results indicate that similar mechanisms to those observed in CAFs may contribute to niche-dependent drug resistance.

The circadian transcription system thus drives the expression of genes that regulate the cell cycle, DNA repair, and thiol production, which are involved in drug sensitivity, indicating that the circadian rhythm may contribute to the efficacy of chemotherapy in cancer patients, with implications for side-effects and patient outcomes, including prognosis (39). New methods are required to understand the status of the circadian rhythm in an individual patient. WNT16B expression has recently been reported to be upregulated in fibroblasts by chemotherapy, and to promote epithelial-mesenchymal transition in neoplastic prostate epithelium through paracrine signaling (40). They also showed that WNT16B promoted the survival of cancer cells after chemotherapy, suggesting that the tumor environment functions as a stromal chemoresistant niche.

\section{Conclusions}

WNT10a is a key molecule in the development of the tumor niche. WNT10a-dependent activation of the tumor niche not only supports the emerging links between the circadian rhythm, oxidative stress and tumor progression at the molecular level, but also alerts us to the potentially adverse effects of artificial light. Further studies are needed to clarify whether WNT10A-Frizzled binding mediates cell proliferation in both endothelial cells and stromal cells. Examining WNT10A receptors and their associated signal transduction pathways may provide valuable insights into the role of circadian 
rhythms in tumor progression. A greater understanding of the complexity of the tumor microenvironment and the role of the tumor niche will lead to further advances in cancer treatment.

Long-term disturbance and disruption of the circadian rhythm contributes to the development of cancer from the preclinical to clinical phases through the evolution of a highly specialized tumor niche (Fig. 6). As expected, WNT10A expression is controlled by a clock gene (41). These results suggest that researchers should consider the relevance of chronobiology based on the results of occupational science related to shift work. Improved understanding of the circadian rhythm will also allow the further development of chronotherapy in cancer patients.

\section{References}

1. Antoni MH, Lutgendorf SK, Cole SW, et al: The influence of bio-behavioural factors on tumour biology: pathways and mechanisms. Nat Rev Cancer 6: 240-248, 2006.

2. Lutgendorf SK, Sood AK and Antoni MH: Host factors and cancer progression: biobehavioral signaling pathways and interventions. J Clin Oncol 28: 4094-4099, 2010.

3. Costa G, Haus E and Stevens R: Shift work and cancer-considerations on rationale, mechanisms, and epidemiology. Scand $\mathrm{J}$ Work Environ Health 36: 163-179, 2010.

4. Kolstad HA: Nightshift work and risk of breast cancer and other cancers - a critical review of the epidemiologic evidence. Scand J Work Environ Health 34: 5-22, 2008.

5. McGregor BA and Antoni MH: Psychological intervention and health outcomes among women treated for breast cancer: a review of stress pathways and biological mediators. Brain Behav Immun 23: 159-166, 2009.

6. Fu L and Lee CC: The circadian clock: pacemaker and tumour suppressor. Nat Rev Cancer 3: 350-361, 2003.

7. Yu EA and Weaver DR: Disrupting the circadian clock: gene-specific effects on aging, cancer, and other phenotypes. Aging (Albany, NY) 3: 479-493, 2011.

8. Borovski T, De Sousa E Melo F, Vermeulen L and Medema JP: Cancer stem cell niche: the place to be. Cancer Res 71: 634-639, 2011.

9. Li L and Xie T: Stem cell niche: structure and function. Annu Rev Cell Dev Biol 21: 605-631, 2005.

10. McAllister SS and Weinberg RA: Tumor-host interactions: a far-reaching relationship. J Clin Oncol 28: 4022-4028, 2010.

11. Lu P, Weaver VM and Werb Z: The extracellular matrix: a dynamic niche in cancer progression. J Cell Biol 196: 395-406, 2012.

12. Provenzano PP, Inman DR, Eliceiri KW, et al: Collagen density promotes mammary tumor initiation and progression. BMC Med 6: 11, 2008.

13. Coghlin C and Murray GI: Current and emerging concepts in tumour metastasis. J Pathol 222: 1-15, 2010.

14. Zhou Y, Yan H, Guo M, et al: Reactive oxygen species in vascular formation and development. Oxid Med Cell Longev 2013: 374963, 2013.

15. Kessenbrock K, Plaks V and Werb Z: Matrix metalloproteinases: regulators of the tumor microenvironment. Cell 141: 52-67, 2010.

16. Kohno K, Uchiumi T, Niina I, et al: Transcription factors and drug resistance. Eur J Cancer 41: 2577-2586, 2005.

17. Kalluri R and Zeisberg M: Fibroblasts in cancer. Nat Rev Cancer 6: 392-401, 2006.

18. Cirri P and Chiarugi P: Cancer-associated-fibroblasts and tumour cells: a diabolic liaison driving cancer progression. Cancer Metastasis Rev 31: 195-208, 2012.
19. Bhowmick NA, Neilson EG and Moses HL: Stromal fibroblasts in cancer initiation and progression. Nature 432: 332-337, 2004.

20. Karnoub AE, Dash AB, Vo AP, et al: Mesenchymal stem cells within tumour stroma promote breast cancer metastasis. Nature 449: 557-563, 2007.

21. Mishra PJ, Mishra PJ, Humeniuk R, et al: Carcinoma-associated fibroblast-like differentiation of human mesenchymal stem cells. Cancer Res 68: 4331-4339, 2008.

22. Quante M, Tu SP, Tomita H, et al: Bone marrow-derived myofibroblasts contribute to the mesenchymal stem cell niche and promote tumor growth. Cancer Cell 19: 257-272, 2011.

23. Liu S, Ginestier C, Ou SJ, et al: Breast cancer stem cells are regulated by mesenchymal stem cells through cytokine networks. Cancer Res 71: 614-624, 2011.

24. Cabarcas SM, Mathews LA and Farrar WL: The cancer stem cell niche - there goes the neighborhood? Int J Cancer 129: 2315-2327, 2011.

25. Yasuniwa Y, Izumi H, Wang KY, et al: Circadian disruption accelerates tumor growth and angio/stromagenesis through a Wnt signaling pathway. PLoS One 5: e15330, 2010

26. Fodde $\mathrm{R}$ and Brabletz $\mathrm{T}$ : Wnt/beta-catenin signaling in cancer stemness and malignant behavior. Curr Opin Cell Biol 19: $150-158,2007$.

27. Pagès F, Galon J, Dieu-Nosjean MC, et al: Immune infiltration in human tumors: a prognostic factor that should not be ignored. Oncogene 29: 1093-1102, 2010.

28. Mantovani A, Germano G, Marchesi F, et al: Cancer-promoting tumor-associated macrophages: new vistas and open questions. Eur J Immunol 41: 2522-2525, 2011.

29. Bateman A: Growing a tumor stroma: a role for granulin and the bone marrow. J Clin Invest 121: 516-519, 2011.

30. Bohring A, Stamm T, Spaich C, et al: WNT10A mutations are a frequent cause of a broad spectrum of ectodermal dysplasias with sex-biased manifestation pattern in heterozygotes. Am J Hum Genet 85: 97-105, 2009.

31. Russell SB, Russell JD, Trupin KM, et al: Epigenetically altered wound healing in keloid fibroblasts. J Invest Dermatol 130: 2489-2496, 2010.

32. Schäfer M and Werner S: Cancer as an overhealing wound: an old hypothesis revisited. Nat Rev Mol Cell Biol 9: 628-638, 2008.

33. Mediavilla MD, Sanchez-Barcelo EJ, Tan DX, Manchester L and Reiter RJ: Basic mechanisms involved in the anti-cancer effects of melatonin. Curr Med Chem 17: 4462-4481, 2010.

34. Torigoe $\mathrm{T}$, Izumi $\mathrm{H}$, Ishiguchi $\mathrm{H}$, et al: Cisplatin resistance and transcription factors. Curr Med Chem Anticancer Agents 5: 15-27, 2005.

35. Tanabe $\mathrm{M}$, Izumi $\mathrm{H}$, Ise $\mathrm{T}$, et al: Activating transcription factor 4 increases the cisplatin resistance of human cancer cell lines. Cancer Res 63: 8592-8595, 2003.

36. Igarashi T, Izumi H, Uchiumi T, et al: Clock and ATF4 transcription system regulates drug resistance in human cancer cell lines. Oncogene 26: 4749-4760, 2007.

37. Miyamoto N, Izumi H, Miyamoto R, et al: Transcriptional regulation of activating transcription factor 4 under oxidative stress in retinal pigment epithelial ARPE-19/HPV-16 cells. Invest Ophthalmol Vis Sci 52: 1226-1234, 2011.

38. Miyamoto N, Izumi H, Noguchi T, et al: Tip60 is regulated by circadian transcription factor clock and is involved in cisplatin resistance. J Biol Chem 283: 18218-18226, 2008.

39. Lévi F, Okyar A, Dulong S, Innominato PF and Clairambault J: Circadian timing in cancer treatments. Annu Rev Pharmacol Toxicol 50: 377-421, 2010.

40. Sun Y, Campisi J, Higano C, et al: Treatment-induced damage to the tumor microenvironment promotes prostate cancer therapy resistance through WNT16B. Nat Med 18: 1359-1368, 2012.

41. Guo B, Chatterjee S, Li L, et al: The clock gene, brain and muscle Arnt-like 1, regulates adipogenesis via Wnt signaling pathway. FASEB J 26: 3453-3463, 2012. 